\title{
Expanding the Chemical Space of Biocompatible Fluorophores: Nanohoops in Cells
}

\author{
Brittany M. White, ${ }^{\circledR}$ Yu Zhao, Taryn E. Kawashima, Bruce P. Branchaud, Michael D. Pluth,*(i) \\ and Ramesh Jasti* ${ }^{*}$
}

Department of Chemistry and Biochemistry, Materials Science Institute, and Institute of Molecular Biology, University of Oregon, Eugene, Oregon 97403, United States

\section{Supporting Information}

ABSTRACT: The design and optimization of fluorescent molecules has driven the ability to interrogate complex biological events in real time. Notably, most advances in bioimaging fluorophores are based on optimization of core structures that have been known for over a century. Recently, new synthetic methods have resulted in an explosion of nonplanar conjugated macrocyclic molecules with unique optical properties yet to be harnessed in a biological context. Herein we report the synthesis of the first aqueous-soluble carbon nanohoop (i.e., a macrocyclic slice of a carbon nanotube prepared via organic synthesis) and demonstrate its bioimaging capabilities in live cells. Moreover, we illustrate that these scaffolds can be easily modified by well-established

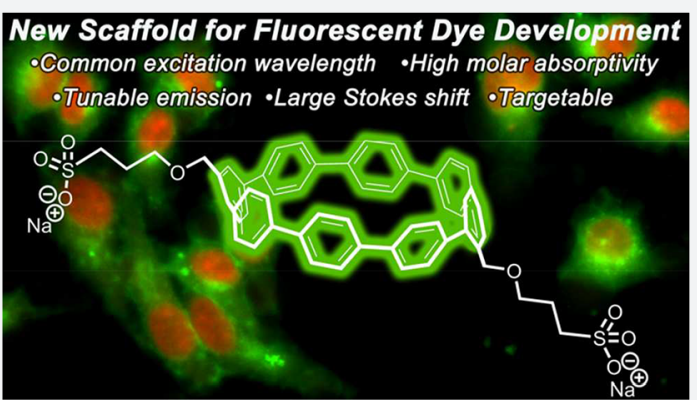
"click" chemistry to enable targeted live cell imaging. This work establishes the nanohoops as an exciting new class of macrocyclic fluorophores poised for further development as novel bioimaging tools.

\section{INTRODUCTION}

Fluorescent molecules have fueled the now widespread use of optical imaging to observe biological processes in living systems. $^{1-3}$ The power of such imaging methods has led to increased interest in identifying new types of dyes, optically active materials, and nanoparticles that have enhanced photophysical properties suitable for multimodal, multiplexed, and super-resolution imaging. ${ }^{4-14}$ Because fluorophores play such a critical role in understanding biological processes, it is somewhat surprising that most advances in small molecule dye technology today rely on structural modifications of scaffolds discovered over a century ago. ${ }^{15}$ For example, the robust Janelia Fluor and some AlexaFluor dyes are structurally modified versions of rhodamine scaffolds discovered 130 years ago. Similarly, commercially available CyDyes, which have found widespread use as probes for targeted live cell imaging, are based off the cyanine core structure synthesized first in 1924 (Figure 1). ${ }^{7}$ Clearly the modification of these core dye scaffolds is still yielding fruitful discoveries (e.g., Janelia Fluor 549); ${ }^{16,17}$ however, fundamentally new types of fluorophore scaffolds could offer advantageous photophysical properties for exploitation in biological contexts. ${ }^{18-21}$ Inspired by this prospect, we report here the first biological studies demonstrating carbon nanohoops, short macrocyclic slices of carbon nanotubes prepared by organic synthesis, as exciting new biocompatible fluorophore scaffolds (Figure 1).

The $[n]$ cycloparaphenylenes $([n]$ CPPs, $n=$ number of benzene rings) are the smallest macrocyclic slices of carbon nanotubes (CNTs). These structures, coined "carbon nanohoops" due to their structural relationship to carbon nanotubes, were intensely pursued synthetic targets for over 70 years before finally succumbing to synthesis in 2008 (Figure 1). ${ }^{22,23}$ Since then, the development of synthetic methods to prepare nanohoops has unveiled several unique, size-dependent photophysical properties that are a direct result of the radially oriented $\pi$-system of this unusual architecture. ${ }^{24-27}$ First, the bending of the $\pi$-system increases delocalization around the hoop due to induction of a small amount of quinoidal character in these strained systems. ${ }^{28}$ Second, the hoop architecture forces neighboring aromatic units to have smaller dihedral angles than in an acyclic oligomeric system due to conformational constraints of the macrocyclic geometry, again leading to increased conjugation. ${ }^{29}$ These two factors together result in a size-dependent fluorescence emission $\left(\lambda_{\text {em }}\right)$ where the HOMO $\rightarrow$ LUMO gap narrows as nanohoop diameter decreases. ${ }^{30}$ Additionally, due to Laporte forbidden HOMO $\rightarrow$ LUMO transitions, all nanohoops share a common absorption maxima $\left(\lambda_{\text {abs }}=340 \mathrm{~nm}\right)$ with high absorption coefficients $(\varepsilon)$ and large effective Stokes shifts ranging from 100 to $200 \mathrm{~nm}$ depending on size. ${ }^{31-34}$ Taken together, the nanohoop scaffold offers the possibility of multiplexed imaging using a single excitation source. Moreover, the nonplanarity of the benzene rings in the nanohoop also leads to better solubility when compared to planar aromatic systems. Lastly, despite molecular strain, nanohoops are only reactive under forcing reaction conditions. ${ }^{35}$ The inherent attributes provided by the nanohoop structure highlight their potential as new

Received: May 30, 2018

Published: August 30, 2018 
Common Organic Dye Scaffolds:
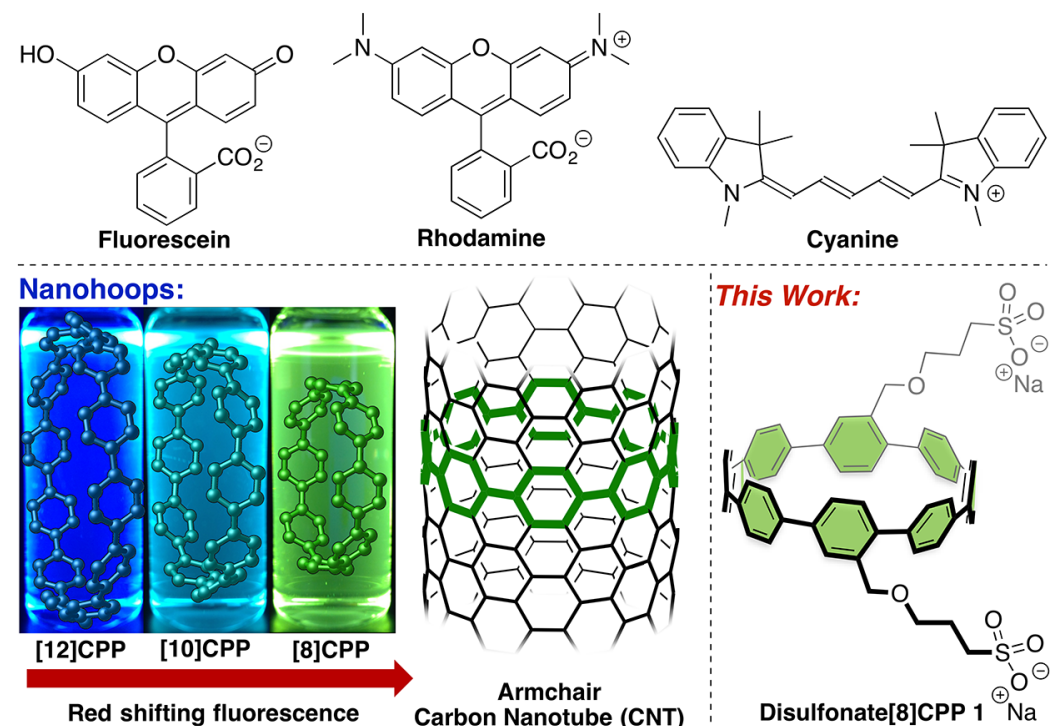

Figure 1. Traditional organic dye scaffolds and the new nanohoop fluorophore scaffold.

Scheme 1. Synthesis of Disulfonate[8]CPP

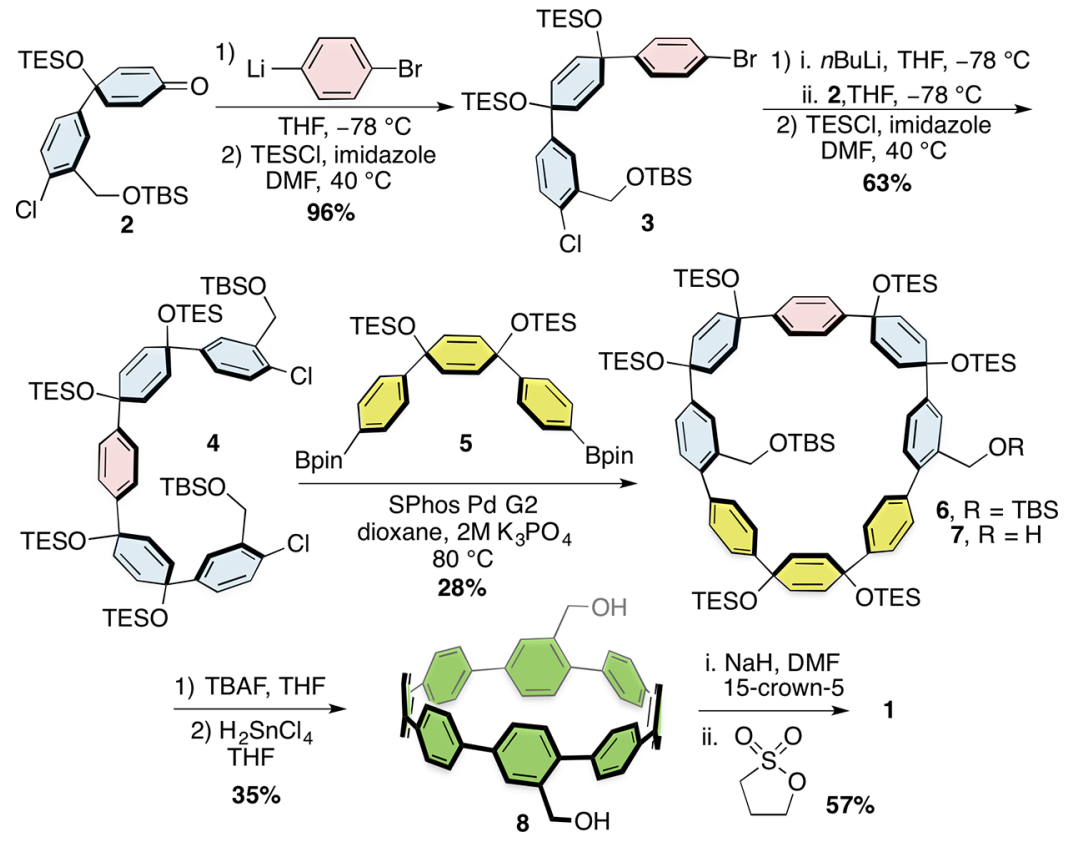

fluorophores for biological imaging. Despite this exciting proposition, to date, there are no reported biological investigations of these small molecular slices of carbon nanotubes. Herein for the first time we report a strategy to prepare an aqueous-soluble nanohoop (1), demonstrate that the desirable optical properties of this scaffold are maintained in aqueous buffer and in live cells, and provide insights into the toxicity and permeability of the nanohoop. We also demonstrate that targeting groups can be easily appended to the nanohoop using copper catalyzed "click" chemistry. This study provides the foundation for the study of nanohoops and their derivatives as an exciting new class of biological imaging tools.

\section{RESULTS AND DISCUSSION}

Numerous studies have documented the promise of carbon nanotubes as biological imaging agents. ${ }^{36}$ Inspired by some of these works, we initially investigated the use of surfactant Pluronic F108 to solubilize the unfunctionalized nanohoops in aqueous media for biological studies-a strategy that has been successful for CNTs. ${ }^{37}$ Although the solubility of the nanohoop increased in the presence of surfactant, cell imaging experiments were plagued by low signal response and aggregation (see the Supporting Information, Figures S1 and $\mathrm{S} 2$ ). This complication prompted the synthesis of $\mathbf{1}$ (Figure 1), a nanohoop functionalized with sulfonate groups to promote solubility in aqueous media. The synthesis of 1 relies on the incorporation of alcohol functional groups into the nanohoop backbone for late stage manipulation (Scheme 1). The 
synthesis begins with the monolithiation of 1,4-dibromobenzene and subsequent nucleophilic addition into ketone 2, followed by protection of the resulting alcohol with triethylsilyl (TES) chloride to give 3 (96\% yield, dr: $>20: 1$ ). Lithiation of 3 followed by nucleophilic addition to a second equivalent of ketone $\mathbf{2}$ and TES protection provided dichloride $\mathbf{4}$ with two tert-butyl dimethylsilyl (TBS) protected benzyl alcohols as reactive handles. Suzuki-Miyaura cross-coupling of $\mathbf{4}$ and diboronate 5 gave macrocycles 6 and 7 in a $28 \%$ combined yield. Global deprotection of both macrocycles followed by $\mathrm{H}_{2} \mathrm{SnCl}_{4}$-promoted reductive aromatization provided benzyl alcohol[8]CPP 8 in $35 \%$ yield. ${ }^{26}$ Deprotonation of the benzyl alcohols with sodium hydride and treatment with 1,3-propane sultone delivered disulfonated[8]CPP (1) in 57\% yield. The building block synthesis outlined here and the oligomeric nature of the nanohoop scaffold should provide access to various sizes of nanohoops, each with unique fluorescent profiles, excited state lifetimes, and Raman signatures due to the size-dependent nature of these properties. ${ }^{29,38-42}$ This structural control is a hallmark of the bottom-up organic synthesis of graphitic materials.

Characterization of the nanohoop with ${ }^{1} \mathrm{H}$ and ${ }^{13} \mathrm{C}\left\{{ }^{1} \mathrm{H}\right\}$ NMR spectroscopy revealed spectra consistent with the expected structure of 1 . Importantly, the nanohoop is completely soluble in DMSO with photophysical properties that are comparable to the parent nanohoop [8]CPP (Figure 2a). Of note, the installation of two sulfonates was sufficient to

\begin{tabular}{|c|c|c|c|c|c|c|c|}
\hline a) & Solvent & $\begin{array}{l}\lambda_{\text {abs }} \\
(\mathrm{nm}) \\
\end{array}$ & $\left(\begin{array}{c}\varepsilon \\
\left(\mathrm{M}^{-1} \mathrm{~cm}^{-1}\right) \\
\end{array}\right.$ & $\begin{array}{c}\lambda_{\text {em }} \\
(\mathrm{nm})\end{array}$ & $\phi_{\mathrm{F}}$ & $\begin{array}{c}\lambda_{\text {em }}-\lambda_{\text {abs }} \\
(\mathrm{nm})\end{array}$ & $\begin{array}{c}\text { Brightness } \\
\left(\varepsilon \times \phi_{\mathrm{F}}\right) \\
\end{array}$ \\
\hline$[8] \mathrm{CPP}^{24}$ & $\mathrm{CHCl}_{3}$ & 340 & $1.0 \times 10^{5}$ & 533 & 0.10 & 193 & $1.0 \times 10^{4}$ \\
\hline 1 & DMSO & 337 & $6.4 \times 10^{4 b}$ & 523 & $0.17^{b}$ & 186 & $1.1 \times 10^{4}$ \\
\hline 1 & PBS Buffera & 328 & $5.8 \times 10^{4 b}$ & 510 & $0.17^{b}$ & 182 & $9.9 \times 10^{3}$ \\
\hline Fluorescein 44 & $\mathrm{EtOH}^{c}$ & 499 & $9.2 \times 10^{4}$ & 540 & 0.97 & 41 & $9.0 \times 10^{4}$ \\
\hline
\end{tabular}
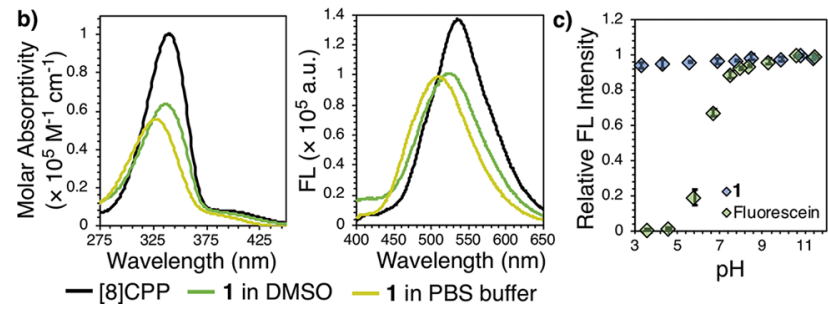

Figure 2. Characterization of disulfonate[8]CPP (1). (a) Summary of nanohoop photophysical properties. (footnote a) Contains $0.1 \%$ SDS. (footnote b) Standard deviation is $<5 \%$ of the measurement $(n=3)$. (footnote c) $0.01 \mathrm{M} \mathrm{KOH}$ in ethanol. (b) $\lambda_{\mathrm{ex}}$ and $\lambda_{\mathrm{em}}$ of $2 \mu \mathrm{M}$ solutions of [8]CPP (black), $\mathbf{1}$ in DMSO (green), and $\mathbf{1}$ in PBS buffer with $0.1 \%$ SDS (yellow). (c) $\mathrm{pH}$ vs fluorescence (FL) intensity of $\mathbf{1}$ and fluorescein in a 1:1 MeOH:100 $\mathrm{mM} \mathrm{KCl}, 100 \mathrm{mM} \mathrm{KOH}$ solution. Error bars represent standard deviation $(n=3)$.

render this nanohoop aqueous-soluble, a result which is consistent with our findings that these nonplanar structures are much more soluble than flat aromatics. Importantly, the photophysical properties of $\mathbf{1}$ are retained in aqueous media (PBS buffer with $0.1 \%$ SDS). Similar to [8]CPP, the absorption maximum for $\mathbf{1}$ is at $328 \mathrm{~nm}$ with a large molar extinction coefficient of $5.8 \times 10^{4} \mathrm{M}^{-1} \mathrm{~cm}^{-1}$. Upon excitation, we observe a bright green fluorescence $\left(\lambda_{\text {em }}=510 \mathrm{~nm}\right)$ with a quantum yield of 0.17 and a large effective Stokes shift of over $180 \mathrm{~nm}$. This is in stark contrast to common fluorophores such as fluorescein that has a Stokes shift of $41 \mathrm{~nm}^{43}$ The fluorescence emission is insensitive to acidic or basic environments $(\mathrm{pH}=3-11)$, which is again in contrast to many common fluorophores (e.g., fluorescein, Figure 2c). Taken together, these findings illustrate that the desirable absorption and emission properties of the nanohoop are not perturbed when the nanohoop scaffold is manipulated to prepare aqueous-soluble versions that can be used for biological studies.

To probe the cytotoxicity of the nanohoop, we treated live HeLa cells with 5, 10, 25, 50, and $100 \mu \mathrm{M}$ solutions of 1 for 2 h. We then monitored cell death using WST-8 formazan reduction (CCK-8 cell assay, Supporting Information, Figure S3). ${ }^{44}$ Nanohoop 1 showed no cytotoxicity at working concentrations of $\leq 10 \mu \mathrm{M}$. Instead, cell death was only observed at concentrations of $25 \mu \mathrm{M}$ and above or with longer incubation times (Supporting Information, Figure S4). We note that more extensive studies of nanohoop toxicology as a function of size, composition, and even encapsulated molecules are warranted in the future. Related studies for other graphitic nanomaterials are often plagued by the inherent heterogeneity of those materials, again highlighting the advantage of the bottom-up synthetic approach for the nanohoops. ${ }^{36}$

Next, using epifluorescence microscopy, we aimed to determine whether $\mathbf{1}$ is cell permeable and whether the fluorescence of the nanohoop is sufficient to generate bright images in live cells. To test this, HeLa cells were treated with a $10 \mu \mathrm{M}$ solution of $\mathbf{1}$ in FBS free DMEM with $0.5 \% \mathrm{DMSO}$ and the nuclear stain NucRed 647 for 1 h (Figure 3E-H). Notably, after incubation and washing, bright green fluorescence from the nanohoop is clearly observed in the cells, which does not colocalize with the nuclear dye. Interestingly, the lack of localization of 1 to specific cellular compartments is consistent with the previously reported localization of calixarenes in Chinese hamster ovary ( $\mathrm{CHO}$ ) cells. ${ }^{45}$ Based on Pearson's correlation coefficients, we observe moderate colocalization to the cytosol (Celltracker Red CMTPX), and lower colocalization to the mitochondria (MitoTracker Red RM) and endoplasmic reticulum (ER-Tracker Red, Supporting Information, Figure S5 and Table S2). ${ }^{46}$ In the absence of 1 (Figure $3 \mathrm{~A}-\mathrm{D})$, no fluorescence was observed in the nanohoop channel confirming that the signal was not due to cellular autofluorescence. Additionally, no significant changes in cell morphology were observed through the differential interference contrast (DIC) channel after incubation with $\mathbf{1}$, confirming a low cytotoxicity of the nanohoop at this concentration.

Encouraged by the robust imaging capabilities of $\mathbf{1}$ in live cells we next sought to demonstrate the flexibility of this new fluorophore scaffold through the preparation of a "clickable" version of the nanohoop. We prepared azide[8]CPP 9 using a scalable synthetic strategy similar to the methods described in Scheme 1 (see the Supporting Information). In this case, we assumed the "clicked" moiety could provide the water solubility. To demonstrate the utility of azide 9 , folate[8]CPP 11 was synthesized using copper catalyzed azide-alkyne cycloaddition (Figure 4a). Folate receptors are known to be highly overexpressed on the surface of many cancer cells. Folic acid $\left(K_{\mathrm{D}}=0.1 \mathrm{nM}\right)$ therefore can be an effective targeting group for imaging of cancer cells and even selective drug delivery. $^{47,48}$

HeLa cells were incubated with a $10 \mu \mathrm{M}$ solution of 11 in FBS free DMEM with $0.1 \%$ DMSO for $2 \mathrm{~h}$ (Figure 4bA,B). The cells were then washed and incubated for $18 \mathrm{~h}$ with FBS- 


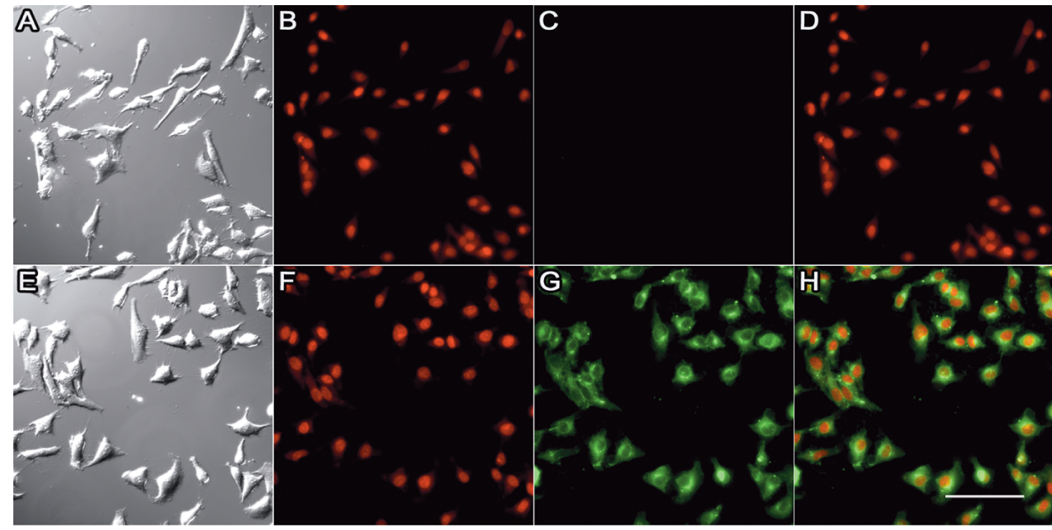

Figure 3. DIC and fluorescent images of live HeLa cells in the absence (A-D) or presence (E-H) of disulfonate[8]CPP (1). (A, E) DIC; (B, F) NucRed live 647 imaged in CY5 channel; (C, G) 1 imaged in DAPI-long-pass channel; and (D, H) merge of the CY5/DAPI-long-pass channel showing no significant colocalization. Scale bar $=100 \mu \mathrm{m}$.

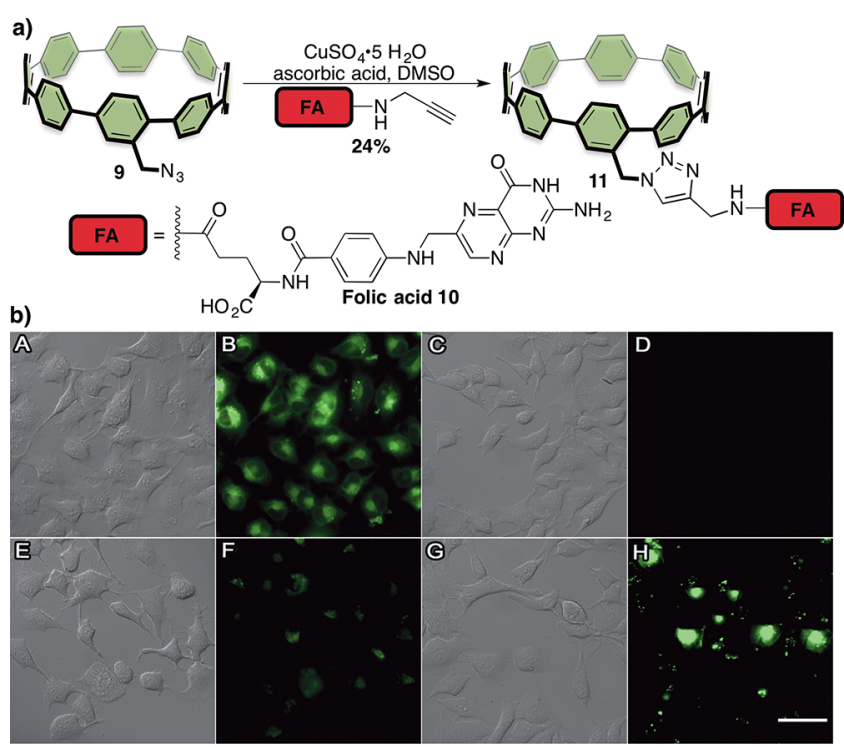

Figure 4. (a) Synthesis of folate-[8]CPP conjugate using copper catalyzed azide-alkyne click chemistry. (b) DIC and fluorescent images of live HeLa cells in the presence of 11 (A, B, E, F) and absence of $11(\mathrm{C}, \mathrm{D}, \mathrm{G}, \mathrm{H})$. As controls cells were treated with folic acid (E, F) and 9 (G, H). (A, C, E, G) DIC channel; (B, D, F, H) DAPI-long-pass channel. Scale bar $=50 \mu \mathrm{m}$.

free DMEM. After the second incubation period and washing, a bright fluorescent emission was observed from the nanohoop. In the absence of 11, no fluorescence is observed confirming that the signal was a result of the emission of $\mathbf{1 1}$ and not cell autofluorescence (Figure $4 \mathrm{bC}, \mathrm{D}$ ). To further support the role of folic acid receptors on the cell uptake of 11, we preincubated cells with free folic acid for $30 \mathrm{~min}$ to saturate the folate receptors. Then, we incubated the cells with a solution containing free folic acid and nanohoop 11. Figure $4 \mathrm{bE}, \mathrm{F}$ shows a marked decrease of cell fluorescence through the nanohoop channel consistent with the folic acid receptor mediated uptake of 11. Furthermore, when cells were treated with 9 nonlocalized fluorescence was observed, which we attribute to aggregation of the azido nanohoop. These results demonstrate that azide[8]CPP 9 can be functionalized with targeting groups and imaged in live cells.

\section{CONCLUSIONS}

These initial studies establish several important points regarding the nanohoop architecture, a growing class of conjugated molecules with radially oriented $\pi$-systems, as a new macrocyclic scaffold for fluorescent dye design. First, sulfonation is a viable strategy to render the nanohoops aqueous-soluble and retain their advantageous photophysical properties. Second, these aqueous-soluble nanohoops can penetrate live cells with minimal cytotoxicity and produce bright fluorescent images. Additionally, our solution measurements show that these materials are $\mathrm{pH}$ insensitive, an important consideration as we begin to develop the wide applicability of this unique molecular structure for intracellular probes where $\mathrm{pH}$ varies dramatically in each cellular compartment. Finally, we established that the nanohoop can be derivatized with targeting groups using "click" chemistry and imaged in live cells. An exciting next step that we are currently pursuing is to establish nanohoops as multiplexed imaging tools utilizing the $\lambda_{\mathrm{abs}}$ shared by all nanohoops and their well resolved and size-dependent fluorescence, singlet lifetimes, and even Raman signatures. For example, based on modern imaging techniques and the synthetic methods available to prepare nanohoops, simultaneous imaging of 20 nanohoops in one experiment is feasible. ${ }^{19,49}$ As a more longterm prospect, we anticipate that the oligomeric nature and unique electron rich cavity of the nanohoop structure can be further engineered to allow for more complex function in biological settings. In conclusion, we have taken an important first step to demonstrate nanohoops as an untapped class of fluorescent dyes that are viable for fluorescent probe development.

\section{ASSOCIATED CONTENT}

\section{S Supporting Information}

The Supporting Information is available free of charge on the ACS Publications website at DOI: 10.1021/acscentsci. 8 b00346.

Experimental procedures and spectral data including NMR spectra, fluorescent images, cell viability, DIC images, quantum yield measurements, molar absorptivity measurements, fluorescence, and absorption and emission spectra (PDF) 


\section{AUTHOR INFORMATION}

\section{Corresponding Authors}

*E-mail: pluth@uoregon.edu.

*E-mail: rjasti@uoregon.edu.

ORCID

Brittany M. White: 0000-0002-0597-7246

Michael D. Pluth: 0000-0003-3604-653X

Ramesh Jasti: 0000-0002-8606-6339

\section{Notes}

The authors declare no competing financial interest.

No unexpected or unusually high safety hazards were encountered.

\section{ACKNOWLEDGMENTS}

Financial support was provided by the National Science Foundation (R.J., CHE-1255219, CHE-1800586), the National Institute of Health (M.D.P., R01GM113030), the Sloan Foundation, the Camille and Henry Dreyfus foundation, and generous startup funds from the University of Oregon. Microscopy support in the UO CAMCOR facility was provided by the NSF (CHE-1531198), and mass spectrometry support was provided by NSF (CHE-1625529).

\section{REFERENCES}

(1) Garland, M.; Yim, J. J.; Bogyo, M. A bright future for precision medicine: advances in fluorescent chemical probe design and their clinical application. Cell Chem. Biol. 2016, 23, 122-136.

(2) Lavis, L. D.; Raines, R. T. Bright ideas for chemical biology. ACS Chem. Biol. 2008, 3, 142-155.

(3) Liu, Z.; Lavis, L. D.; Betzig, E. Imaging live-cell dynamics and structure at the single-molecule level. Mol. Cell 2015, 58, 644-659.

(4) Jones, K. A.; Porterfield, W. B.; Rathbun, C. M.; McCutcheon, D. C.; Paley, M. A.; Prescher, J. A. Orthogonal luciferase-luciferin pairs for bioluminescence imaging. J. Am. Chem. Soc. 2017, 139, 2351-2358.

(5) Michie, M. S.; Gotz, R.; Franke, C.; Bowler, M.; Kumari, N.; Magidson, V.; Levitus, M.; Loncarek, J.; Sauer, M.; Schnermann, M. J. Cyanine Conformational restraint in the far-red range. J. Am. Chem. Soc. 2017, 139, 12406-12409.

(6) Chattopadhyay, P. K.; Gaylord, B.; Palmer, A.; Jiang, N.; Raven, M. A.; Lewis, G.; Reuter, M. A.; Rahman, A. K. M. N. U.; Price, D. A.; Betts, M. R.; Roederer, M. Brilliant violet fluorophores: A new class of ultrabright fluorescent compounds for immunofluorescence experiments. Cytometry, Part A 2012, 81a, 456-466.

(7) Lavis, L. D. Chemistry is dead. Long live chemistry! Biochemistry 2017, 56, 5165-5170.

(8) Xue, L.; Karpenko, I. A.; Hiblot, J.; Johnsson, K. Imaging and manipulating proteins in live cells through covalent labeling. Nat. Chem. Biol. 2015, 11, 917-923.

(9) Rodriguez, E. A.; Campbell, R. E.; Lin, J. Y.; Lin, M. Z.; Miyawaki, A.; Palmer, A. E.; Shu, X. K.; Zhang, J.; Tsien, R. Y. The growing and glowing toolbox of fluorescent and photoactive proteins. Trends Biochem. Sci. 2017, 42, 111-129.

(10) Kairdolf, B. A.; Qian, X. M.; Nie, S. M. Bioconjugated nanoparticles for biosensing, in vivo imaging, and medical diagnostics. Anal. Chem. 2017, 89, 1015-1031.

(11) Butkevich, A. N.; Lukinavicius, G.; D’Este, E.; Hell, S. W. Cellpermeant large Stokes shift dyes for transfection-free multicolor nanoscopy. J. Am. Chem. Soc. 2017, 139, 12378-12381.

(12) Lee, H. L. D.; Lord, S. J.; Iwanaga, S.; Zhan, K.; Xie, H. X.; Williams, J. C.; Wang, H.; Bowman, G. R.; Goley, E. D.; Shapiro, L.; Twieg, R. J.; Rao, J. H.; Moerner, W. E. Superresolution Imaging of targeted proteins in fixed and living cells using photoactivatable organic fluorophores. J. Am. Chem. Soc. 2010, 132, 15099-15101.
(13) Banala, S.; Maurel, D.; Manley, S.; Johnsson, K. A caged, localizable rhodamine derivative for superresolution microscopy. ACS Chem. Biol. 2012, 7, 289-293.

(14) Chozinski, T. J.; Gagnon, L. A.; Vaughan, J. C. Twinkle, twinkle little star: Photoswitchable fluorophores for super-resolution imaging. FEBS Lett. 2014, 588, 3603-3612.

(15) Lavis, L. D.; Raines, R. T. Bright building blocks for chemical biology. ACS Chem. Biol. 2014, 9, 855-866.

(16) Grimm, J. B.; English, B. P.; Chen, J. J.; Slaughter, J. P.; Zhang, Z. J.; Revyakin, A.; Patel, R.; Macklin, J. J.; Normanno, D.; Singer, R. H.; Lionnet, T.; Lavis, L. D. A general method to improve fluorophores for live-cell and single-molecule microscopy. Nat. Methods 2015, 12, 244-250.

(17) Grimm, J. B.; Muthusamy, A. K.; Liang, Y.; Brown, T. A.; Lemon, W. C.; Patel, R.; Lu, R.; Macklin, J. J.; Keller, P. J.; Ji, N.; Lavis, L. D. A general method to fine-tune fluorophores for live-cell and in vivo imaging. Nat. Methods 2017, 14, 987-994.

(18) Qu, J. Q.; Kohl, C.; Pottek, M.; Mullen, K. Ionic perylenetetracarboxdiimides: Highly fluorescent and water-soluble dyes for biolabeling. Angew. Chem., Int. Ed. 2004, 43, 1528-1531.

(19) Hu, F. H.; Zeng, C.; Long, R.; Miao, Y. P.; Wei, L.; Xu, Q. Z.; Min, W. Supermultiplexed optical imaging and barcoding with engineered polyynes. Nat. Methods 2018, 15, 194-200.

(20) Lin, H. A.; Sato, Y.; Segawa, Y.; Nishihara, T.; Sugimoto, N.; Scott, L. T.; Higashiyama, T.; Itami, K. A water-soluble warped nanographene: synthesis and applications for photoinduced cell death. Angew. Chem., Int. Ed. 2018, 57, 2874-2878.

(21) Wan, H.; Yue, J. Y; Zhu, S. J; Uno, T.; Zhang, X. D; Yang, Q. L; Yu, K.; Hong, G. S; Wang, J. Y; Li, L. L; Ma, Z. R; Gao, H. P; Zhong, Y. T; Su, J.; Antaris, A. L; Xia, Y.; Luo, J.; Liang, Y. Y; Dai, H. J. A bright organic NIR-II nanofluorophore for three-dimensional imaging into biological tissues. Nat. Commun. 2018, 9, 1171.

(22) Golder, M. R.; Jasti, R. Syntheses of the smallest carbon nanohoops and the emergence of unique physical phenomena. Acc. Chem. Res. 2015, 48, 557-566.

(23) Jasti, R.; Bhattacharjee, J.; Neaton, J. B.; Bertozzi, C. R. Synthesis, characterization, and theory of [9]-, [12]-, and [18]cycloparaphenylene: carbon nanohoop structures. J. Am. Chem. Soc. 2008, 130, 17646-17647.

(24) Scott, L. T. Conjugated belts and nanorings with radially oriented p orbitals. Angew. Chem., Int. Ed. 2003, 42, 4133-4135.

(25) Darzi, E. R.; Sisto, T. J.; Jasti, R. Selective syntheses of [7][12] cycloparaphenylenes using orthogonal Suzuki-Miyaura crosscoupling reactions. J. Org. Chem. 2012, 77, 6624-6628.

(26) Patel, V. K.; Kayahara, E.; Yamago, S. Practical synthesis of [n]cycloparaphenylenes $(\mathrm{n}=5,7-12)$ by $\mathrm{H} 2 \mathrm{SnCl} 4$-mediated aromatization of 1,4-dihydroxycyclo-2,5-diene precursors. Chem. Eur. J. 2015, 21, 5742-5749.

(27) Segawa, Y.; Yagi, A.; Matsui, K.; Itami, K. Design and synthesis of carbon nanotube segments. Angew. Chem., Int. Ed. 2016, 55, 51365158.

(28) Pena-Alvarez, M.; Qiu, L. L.; Taravillo, M.; Baonza, V. G.; Delgado, M. C. R.; Yamago, S.; Jasti, R.; Navarrete, J. T. L.; Casado, J.; Kertesz, M. From linear to cyclic oligoparaphenylenes: electronic and molecular changes traced in the vibrational Raman spectra and reformulation of the bond length alternation pattern. Phys. Chem. Chem. Phys. 2016, 18, 11683-11692.

(29) Darzi, E. R.; Jasti, R. The dynamic, size-dependent properties of [5]-[12] cycloparaphenylenes. Chem. Soc. Rev. 2015, 44, 6401-6410. (30) Adamska, L.; Nayyar, I.; Chen, H.; Swan, A. K.; Oldani, N.; Fernandez-Alberti, S.; Golder, M. R.; Jasti, R.; Doorn, S. K.; Tretiak, S. Self-trapping of excitons, violation of Condon approximation and efficient fluorescence in conjugated cycloparaphenylenes. Nano Lett. 2014, 14, 6539-6546.

(31) Iwamoto, T.; Watanabe, Y.; Sakamoto, Y.; Suzuki, T.; Yamago, $\mathrm{S}$. Selective and random syntheses of $[\mathrm{n}]$ cycloparaphenylenes $(\mathrm{n}=8-$ 13) and size dependence of their electronic properties. J. Am. Chem. Soc. 2011, 133, 8354-8361. 
(32) Darzi, E. R.; Hirst, E. S.; Weber, C. D.; Zakharov, L. N.; Lonergan, M. C.; Jasti, R. Synthesis, properties, and design principles of donor-acceptor nanohoops. ACS Cent. Sci. 2015, 1, 335-342.

(33) Kuwabara, T.; Orii, J.; Segawa, Y.; Itami, K. Curved oligophenylenes as donors in shape-persistent donor-acceptor macrocycles with solvatofluorochromic properties. Angew. Chem., Int. Ed. 2015, 54, 9646-9649.

(34) Van Raden, J. M.; Darzi, E. R.; Zakharov, L. N.; Jasti, R. Synthesis and characterization of a highly strained donor-acceptor nanohoop. Org. Biomol. Chem. 2016, 14, 5721-5727.

(35) Sisto, T. J.; Zakharov, L. N.; White, B. M.; Jasti, R. Towards piextended cycloparaphenylenes as seeds for CNT growth: investigating strain relieving ring-openings and rearrangements. Chem. Sci. 2016, 7, 3681-3688.

(36) Hong, G. S.; Diao, S. O.; Antaris, A. L.; Dai, H. J. Carbon nanomaterials for biological imaging and nanomedicinal therapy. Chem. Rev. 2015, 115, 10816-10906.

(37) Cherukuri, P.; Gannon, C. J.; Leeuw, T. K.; Schmidt, H. K.; Smalley, R. E.; Curley, S. A.; Weisman, R. B. Mammalian pharmacokinetics of carbon nanotubes using intrinsic near-infrared fluorescence. Proc. Natl. Acad. Sci. U. S. A. 2006, 103, 18882-18886.

(38) Segawa, Y.; Fukazawa, A.; Matsuura, S.; Omachi, H.; Yamaguchi, S.; Irle, S.; Itami, K. Combined experimental and theoretical studies on the photophysical properties of cycloparaphenylenes. Org. Biomol. Chem. 2012, 10, 5979-5984.

(39) Hines, D. A.; Darzi, E. R.; Jasti, R.; Kamat, P. V. Carbon nanohoops: excited singlet and triplet behavior of [9]- and [12]cycloparaphenylene. J. Phys. Chem. A 2014, 118, 1595-1600.

(40) Chen, H.; Golder, M. R.; Wang, F.; Jasti, R.; Swan, A. K. Raman spectroscopy of carbon nanohoops. Carbon 2014, 67, 203-213.

(41) Pena-Alvarez, M.; Burrezo, P. M.; Kertesz, M.; Iwamoto, T.; Yamago, S.; Xia, J. L.; Jasti, R.; Navarrete, J. T. L.; Taravillo, M.; Baonza, V. G.; Casado, J. Properties of sizeable [n]cycloparaphenylenes as molecular models of single-wall carbon nanotubes elucidated by raman spectroscopy: structural and electron-transfer responses under mechanical stress. Angew. Chem., Int. Ed. 2014, 53, 7033-7037.

(42) Yamago, S.; Watanabe, Y.; Iwamoto, T. Synthesis of [8]cycloparaphenylene from a square-shaped tetranuclear platinum complex. Angew. Chem., Int. Ed. 2010, 49, 757-759.

(43) Seybold, P. G.; Gouterman, M.; Callis, J. Calorimetric, photometric and lifetime determinations of fluorescence yields of fluorescein dyes. Photochem. Photobiol. 1969, 9, 229-242.

(44) Dojindo Molecular Technologies, Inc. Cell Counting Kit-8: Technical Manual. https://www.dojindo.com/TechnicalManual/ Manual_CK04.pdf (accessed Aug 19, 2018).

(45) Lalor, R.; Baillie-Johnson, H.; Redshaw, C.; Matthews, S. E.; Mueller, A. Cellular uptake of a fluorescent calix[4]arene derivative. J. Am. Chem. Soc. 2008, 130, 2892-2893.

(46) Dunn, K. W.; Kamocka, M. M.; McDonald, J. H. A practical guide to evaluating colocalization in biological microscopy. Am. J. Physicol. Cell Physiol. 2011, 300, C723-C742.

(47) Consoli, G. M. L.; Granata, G.; Fragassi, G.; Grossi, M.; Sallese, M.; Geraci, C. Design and synthesis of a multivalent fluorescent folate-calix[4]arene conjugate: cancer cell penetration and intracellular localization. Org. Biomol. Chem. 2015, 13, 3298-3307.

(48) Consoli, G. M. L.; Granata, G.; Geraci, C. Design, synthesis, and drug solubilising properties of the first folate-calixarene conjugate. Org. Biomol. Chem. 2011, 9, 6491-6495.

(49) Huang, T.; Phelps, C.; Wang, J.; Lin, L. J.; Bittel, A.; Scott, Z.; Jacques, S.; Gibbs, S. L.; Gray, J. W.; Nan, X. L. Simultaneous multicolor single-molecule tracking with single-laser excitation via spectral imaging. Biophys. J. 2018, 114, 301-310. 